Voix et Images

volxetimages

\title{
Variations sur le thème de l'engagement
}

ANNE CAUMARTIN

Volume 43, numéro 1 (127), automne 2017

URI : https://id.erudit.org/iderudit/1043154ar

DOI : https://doi.org/10.7202/1043154ar

Aller au sommaire du numéro

Éditeur(s)

Université du Québec à Montréal

ISSN

0318-9201 (imprimé)

1705-933X (numérique)

Découvrir la revue

Citer ce compte rendu

CAUMARTIN, A. (2017). Compte rendu de [Variations sur le thème de

l'engagement]. Voix et Images, 43(1), 113-118. https://doi.org/10.7202/1043154ar d'utilisation que vous pouvez consulter en ligne.

https://apropos.erudit.org/fr/usagers/politique-dutilisation/ 


\title{
E S S A I S / ÉT U D E S
}

\author{
Variations sur le thème de l'engagement \\ $+++$
}

ANNE CAUMARTIN

Collège militaire royal de Saint-Jean

Avec l'étude La résistance en héritage ${ }^{1}$, Rachel Nadon porte un regard neuf sur ce lieu incontournable de la littérature québécoise qu'a été Liberté. Un rapide recensement des études parues sur le sujet indique que la critique s'est surtout intéressée aux débuts de la revue, l'examinant le plus souvent jusqu'aux années 1980, portant aussi une attention particulière à la figure de l'écrivain et aux représentations de l'intellectuel qui en émanent. Ce geste critique a construit une sorte de glorification des débuts et a scellé un âge d'or de la revue, discréditant en creux ses suites plus éloignées du politique, et étouffant presque l'avenir de Liberté avant même qu'il n'advienne, comme si toute nouvelle orientation ne pouvait qu'être vite dépassée, tout projet différant du passé semblant d'emblée nul et non avenu. Nadon, en montrant son intérêt critique pour la période actuelle (2006-2011, avec la nouvelle direction de Pierre Lefebvre jusqu'à la veille de la refonte de la présentation visuelle de Liberté), ouvre en quelque sorte la revue à son avenir.

La démarche de l'auteure tient en une étude, soignée et intelligente, de la filiation. Elle revient sur les collaborateurs de la première heure, sur les diverses figures tutélaires qui ont modelé la revue pour examiner comment s'est opéré l'héritage. C'est donc à une pensée historicisée qu'on a affaire ici alors qu'est présenté le travail de Liberté sous la direction de Lefebvre, faisant de cette nouvelle équipe une génération de collaborateurs avec des influences et des orientations particulières. Si le mot "génération» peut parfois être reçu avec circonspection, il faut convenir qu'il est ici davantage qu'une notion commode. On associe trop souvent cette idée à celle des âges, qui eux, supposément, forceraient la cohérence intellectuelle - ce qui a déjà été vrai lorsque la cohérence intellectuelle au Québec était principalement déterminée par l'éducation et le milieu social. Les facteurs de cette cohérence sont aujourd'hui multiples. Les générations ne se réunissent plus autant autour d'une année de naissance, mais il n'en reste pas moins qu'elles existent dans la mesure où, à un moment, des individus se reconnaissent par une vision du monde, une vision de la littérature en l'occurrence, et une façon de s'y insérer en fonction d'influences

1 Rachel Nadon, La résistance en héritage. Le discours culturel des essayistes de Liberté (2006-2011), Montréal, Nota bene, coll. «Prémices», 2016, 224 p. 
choisies. C'est d'ailleurs la beauté des filiations intellectuelles: elles peuvent être choisies. Choisies horizontalement si on peut dire, comme réseau d'individus qui témoignent semblablement de leur temps, et qui y agissent aussi semblablement. Réside là la force de penser en communauté, de savoir qui sont ses alliés. Elles sont choisies verticalement aussi par l'élection de prédécesseurs inspirants qui ont tracé une voie, par celle d'un lieu qui fournit un cadre à cette parole commune - ce qualificatif étant bien relatif, car on ne peut croire que chacun des membres fait une profession de foi délibérée et explicite. Le sentiment d'appartenance, au groupe comme au lieu, peut être quelque chose de diffus, de difficilement nommable, mais c'est en somme ce pouvoir d'attraction intellectuel qui supporte l'idée de génération, qui fait qu'un témoin est passé d'un moment à un autre et que le jeu d'influences, audelà de toutes les précautions rhétoriques d'usage lorsqu'on aborde un concept, est bien réel. Après tout, les vignettes Wikipédia fournissent bien sous certaines figures intellectuelles les sous-titres «influencé par» et «a influencé». Et si Wikipédia le dit, $c^{\prime}$ est que ce doit être vrai.

Certes, la réorientation de Liberté en 2006 a pu mettre à mal l'idée d'une filiation au sein de la revue, la cohésion des groupes intellectuels dans la période contemporaine étant mise en question par certaines figures tutélaires de la revue, dont Jacques Godbout. Lors d'une table ronde en octobre 2014 où il était question de façon générale de l'état de la littérature québécoise contemporaine mais aussi, en particulier, de la transformation de la revue Liberté, Godbout affirmait sans ambages que «le projet littéraire, maintenant, n'est plus générationnel ${ }^{2}$ ». L'auteur du Murmure marchand estime que la logique de marché est ce qui caractérise maintenant les manifestations littéraires: la littérature québécoise contemporaine n'aurait plus de rôle référentiel, elle est maintenant considérée comme un divertissement. Les publications se multiplient de façon étourdissante, la durée de vie d'une œuvre tient à peine en une saison, les auteurs sont des individus qui «ne se réclament d'aucune génération particulière et d'aucune aventure esthétique». Pour preuve, les concepts "publicitaires» de littérature migrante ou LGBT font figure d'avant-gardes dans les médias, et une «littérature-monde de toutes les langues représente le nouvel horizon des jeunes auteurs». En somme, puisque «le paradigme des générations pour la mise en récit de la littérature [lui] semble fort peu pertinent", il serait difficile de croire qu'une communauté critique, qui s'inscrit tout autant dans le champ littéraire, puisse corollairement mettre en scène une pensée articulée par une logique générationnelle.

Pourtant, à Liberté, il existe une tradition de l'ouverture aux points de vue divergents pour autant qu'ils cherchent le même horizon, créant de fait une communauté de pensée. Godbout se plaît d'ailleurs à rappeler son bon mot qui, à ceux qui voyaient cette revue comme une chapelle, répondait que Liberté était une "cathédrale» - il y a de la place pour tout le monde. S'il y a une cohérence à Liberté, elle ne

2 Jacques Godbout, panéliste de la table ronde «La question des générations dans la pensée de la littérature contemporaine», librairie Le port de tête, Montréal, 3 octobre 2014. En ligne: http://radiospirale.org/ capsule/la-question-des-generations-dans-la-pensee-de-la-litterature-contemporaine (page consultée le 27 octobre 2017). 
tient pas tant aux propos, qu'ils soient nationaux ou idéologiques, mais au mouvement même de son ouverture, de son accueil, qui sert essentiellement de relais entre la littérature et la place publique.

Plus prosaïquement qu'à une cathédrale, c'est à la taverne que André Belleau associait Liberté, image qui ne s'est jamais réellement effacée. On y entre, on y circule comme bon nous semble. On y parle fort, puis on retourne dans le monde. C'est à ce mouvement que l'équipe de 2006 a en quelque sorte voulu se rattacher en rapprochant la fonction de la publication de celle de l'agora. Cette affiliation claire distingue l'entreprise de 2006 des autres projets de revues du début du millénaire, qui parfois s'essoufflent rapidement malgré leurs appels insistants mais épars aux nécessaires solidarités. Au contraire, la version de Liberté que présente Rachel Nadon fait la proposition d'un engagement structuré par la perspective historique de la revue. Ce n'est pas celui que montrait Liberté en pleine Révolution tranquille; il ne s'agit plus d'appeler un nouvel ordre, laïque et axé sur la question nationale, mais d'interroger les impératifs du néolibéralisme et de secouer une culture qui est trop souvent tentée par une logique économique et utilitariste. L'objet de préoccupation diffère, mais le lien - l'attention - à la société est assez semblable. S'il y a une désaffiliation - une distinction, une trahison - des prédécesseurs, elle se trouve en regard de la période qu'on pourrait nommer «du repli poétique» dans les années 1990. Le référendum de 1980 a eu des répercussions dans le champ culturel qui se sont lentement cristallisées pour sceller un discours dépolitisé au cours de la décennie suivante, les dossiers thématiques à caractère socioculturel se faisant rares pendant une quinzaine d'années à Liberté.

L'ouvrage de Nadon met bien en évidence la préoccupation renouvelée envers les considérations sociales de l'équipe de 2006 par son titre même. L'idée de résistance suppose à la fois un souci devant un état du monde et les différents objets d'adversité à circonscrire, et la promotion d'un idéal que peut présenter le discours littéraire. L'auteure montre trois temps de cette démarche à travers les modes discursifs que sont la ferveur, la lutte et la déraison, présentés par Olivier Kemeid un peu avant l'éditorial «Assoiffés de sens», paru en 2006 dans le numéro consacré à «la résistance culturelle» ${ }^{3}$. L'intention de Nadon est de situer cette nouvelle prise de parole en examinant le lien entretenu avec l'institution qu'est devenue Liberté au fil du temps, en examinant aussi la description de cette nouvelle orientation, tout comme les enjeux discursifs que celle-ci expose.

Le premier chapitre est consacré à la scénographie des éditoriaux de la nouvelle équipe. À partir de différents textes d'Olivier Kemeid, on montre comment se formule l'appel à l'engagement dans la nouvelle mouture de la revue. Ces textes deviennent programmatiques, ils remplissent en quelque sorte une fonction de manifeste en établissant les jalons de la scène d'énonciation qui se donne à reconstruire. L'auteure explique bien les paramètres de la scène englobante qu'est le champ des

3 Olivier Kemeid, «Pour une nouvelle trahison des clercs», Liberté, vol. XLVII, n 2, mai 2005, p. 111-121, dossier «L'intellectuel sans domicile fixe». Ces idées sont reprises rapidement pour représenter l'ensemble de la nouvelle démarche de la revue: [Comité de rédaction], "Assoiffés de sens», Liberté, vol. XLVIII, nº 3, septembre 2006, p. 3-5, dossier «La résistance culturelle». 
revues, non par les objets de discours qu'elle susciterait, mais plutôt par ce que le type de discours qu'elle permet crée et limite à la fois. Une fois ce passage contextuel franchi, on arrive à une explication plus intéressante et pertinente qui est celle de la scénographie ou, en d'autres mots, à un commentaire sur le repositionnement du discours de Liberté elle-même. En exposant le texte d'intention de Liberté en fonction de ce troisième élément de la topographie du discours chez Dominique Maingueneau, Nadon montre la tension qui s'installe dans l'historicisation de la revue. Un groupe, lorsqu'il s' «empare» d'une revue, «induit une comparaison» avec les manifestations précédentes et «réactive la mémoire du lieu» (41). C'est donc la fonction des textes d'intention des revues qui est appelée à l'évaluation, ces derniers étant conçus comme des éléments de démarcation générationnelle presque indiscutables. Nadon parcourt rapidement la fortune de Liberté en fonction des différents comités de direction qui se sont succédé. Il faut noter au passage que l'auteure réalise une synthèse de l'histoire assez vaste d'une institution littéraire, mais plusieurs éléments critiques intéressants sont repoussés dans les notes de bas de page alors qu'ils mériteraient d'être intégrés au corps de la démonstration. À titre d'exemple, le commentaire de Laurent Mailhot dans un article paru en 1991 qui déplorait déjà l'atténuation de la verve des essayistes de Liberté, qui s'égarent parfois «dans la dérision systématique, dans les polémiques artificielles ou, au contraire, dans une apparente démission éditoriale» (40-41). Le survol qu'effectue Nadon montre d'ailleurs qu'au fil de ses transformations, la revue apparaît comme «scène "héritée" » (42), le lieu devenant un enjeu, et ce même hors des textes d'intention qui servent en quelque sorte de figures d'insistance ou de matériau plus facilement manipulable pour les études critiques.

Le second chapitre, intitulé " "Penser par soi-même" : une communauté d'héritiers dévoyés", présente quant à lui la façon dont cette génération à Liberté s'est située par rapport à ses prédécesseurs. La figure de l'héritier qu'incarnent les collaborateurs de cette période se constitue par «l'activation d'un signe autonome qui fonde une interprétation du texte» (67), façon dont apparaît le geste symbolique de la réception d'un legs culturel au sein de cette institution. Déchirés que sont ces collaborateurs entre le devoir de conquérir un héritage et l'obligation de le trahir, leur geste d'affiliation se fait dans la construction toute rhétorique d'une image de soi. Nadon choisit des exemples éloquents, que ce soit de façon large, dans le champ littéraire, ou au plus près de la revue. Le numéro consacré à Arthur Buies, en permettant des lectures du lien entre politique et littérature, donne l'occasion d'afficher des valeurs - le courage, la combativité, l'insubordination intellectuelle - dont Liberté veut faire la promotion et qui, en retour, permettent de la caractériser. Plus directement, le legs se fait aussi par rapport aux équipes précédentes. Le débat qui s'est tenu entre Olivier Kemeid et Jacques Godbout aussi bien dans Le Devoir que dans Liberté permet de redéfinir la position de la revue. Alors que Godbout représente l'intention initiale de Liberté, et bien que l'équipe de 2006 veuille se rapprocher de la pratique littéraire proche de la société qui était caractéristique de cette intention, des révisions s'imposent. La nostalgie de Godbout veut établir la période de fondation comme l'époque où il y avait «une cohésion extraordinaire et un rêve partagé dans la société» (78). Ce rêve est intimement lié à l'accession au pouvoir du Parti québécois en 1976, ce que critique Kemeid, qui voit moins là le désir d'une libération des 
anciennes contraintes que la simple mise en place d'un nouveau pouvoir. Les effets de l'immigration auraient pour Godbout anéanti les démarches qui consolidaient la culture québécoise puisque le présent, «inquiétant et complexe», "ne porte plus les traces du passé» (81); pour Kemeid, plutôt, et pour la nouvelle équipe de Liberté, «le passé est à relire et à réécrire; c'est la disparition de la culture de l'espace public qui pose problème» (81). Si le but reste le même pour ces deux époques de Liberté, la nuance impose un changement de méthode qu'on imagine bien une trahison pour Godbout. La réécriture de l'histoire et le rapport à la culture se définissent maintenant à partir des marges de la société québécoise, et le devoir de mémoire est essentiellement une réinvention des héritages qui refuse le cantonnement national pour intégrer les contributions diverses à la culture québécoise. Enfin, Nadon aborde la forme de l'hommage comme un procédé de resituation générationnelle, voire de trahison du legs. Les numéros bilans ${ }^{4}$ scellent l'espace de la connaissance; l'héritage se donne comme en un bloc qu'il convient de ciseler pour mieux en voir les constituants. La rétrospective, après avoir opéré une sélection, après avoir proposé fortement aussi une manière de lire ses prédécesseurs, crée un pont, voire une uniformisation, entre le passé et le présent. Ces numéros, plus que de simples hommages, «montrent comment l'héritier se construit à partir des bilans critiques et avec une chronotopie de l'“après", de la coupure, du retour et de l'adieu» (99). C'est en somme - c'est moi qui infère ici - une façon d'utiliser le passé puis de le confiner à un temps révolu pour pouvoir occuper librement l'espace discursif actuel.

Le dernier chapitre montre Liberté en lectrice. Les figures tutélaires d'écrivains remplissent une fonction d'autodéfinition, et Nadon observe de quelles manières les écrivains et les œuvres citées s'inscrivent dans les numéros, de même que les enjeux (idéologiques, critiques, légitimants) que cela suscite. Au cours des cinq années examinées, seulement quatre écrivains sont mis en valeur dans trois numéros: René Char/Hervé Bouchard, Arthur Buies et Nikos Kachtitsis. Ils ne constituent pas pour autant des ancrages fondamentaux de la pensée littéraire de la nouvelle équipe comme peuvent l'être Aquin et Borduas. Nadon mène une analyse comparative minutieuse entre les époques: ces deux "pères» ne sont pas convoqués jadis et maintenant de la même manière, n'ont pas la même signification. Ils disaient la reconnaissance d'un apprentissage mené par les essayistes; ils sont maintenant appelés pour montrer le lien qu'ils entretenaient avec Liberté, son histoire et les idéaux qu'elle veut mettre au jour, permettant une économie du discours en véhiculant un imaginaire et une axiologie qui leur sont propres mais qu'on peut présenter à nouveau sous un angle politique:

L'œuvre n'est pas citée, analysée et étudiée pour elle-même, pour son caractère national, sa complexité formelle ou selon un angle de lecture qui en révélerait un aspect insoupçonné. C'est plutôt à partir d'une refiguration de l'écrivain choisi, des valeurs qui lui sont attribuées, que sont lus les enjeux culturels et politiques. (129)

Ainsi, Aquin permet de concevoir le devenir de la littérature et de la société québécoises, d'une part en fonction d'une tradition d'achoppement de lecture et, d'autre

4 Il s'agit de numéros qui cherchent à aborder sous différents angles des sujets parfois sensibles. 
part, en fonction d'un héritage de la revue, comme si Aquin, objet privilégié de la première Liberté, accordait quelque droit d'initiés à même de révéler une vérité sur le rôle social de cette figure d'écrivain. La fonction Aquin remplacerait en quelque sorte la nécessité de lire l'œuvre, mais sa représentation dans le champ littéraire supporterait la mobilisation sociale que veut promouvoir la nouvelle Liberté par l'intermédiaire de la littérature. De la même manière, les figures de Borduas et de Refus global sont comparées, et Nadon indique leur utilité plus cruciale dans la période actuelle de la revue. Si on accorde moins d'importance au travail pictural de Borduas, une plus grande place est dévolue au manifeste et à la transition qu'il a appelée. Recourir au manifeste sert l'exposition d'une éthique de la littérature, une façon de montrer «la nécessaire et éclatante opposition à l'immobilisme ambiant, aux idées reçues et à la servitude du pouvoir» (149). Cela sert aussi de contre-exemple. Au fil des décennies, le Québec aurait perdu de vue l'héritage du manifeste et de Liberté sur les possibles qui auraient pu advenir si les acteurs culturels - ceux de la littérature au premier rang - avaient été plus vigilants. En somme, ces "pères» servent dans la mesure où ils sont ramenés à l'actualité et aux problèmes socioculturels qu'il convient de signaler par le discours littéraire. C'est à travers eux que se rend visible le geste de résistance nécessaire.

C'est un portrait assez complet que brosse Rachel Nadon de la situation de Liberté entre 2006 et 2011, de la posture d'héritiers de ses principaux collaborateurs et de sa direction. Quel héritage cette génération est-elle prête à léguer? Pour ce qui est de la revue elle-même, l'année 2012 a été le moment d'une refonte complète de sa présentation visuelle comme de son organisation, mais la revue demeure fidèle à la directive de l'attention au monde et de l'engagement explicite établie dès 2006. Plus largement, il est permis de croire que Liberté a débordé de son cadre, certains collaborateurs importants de son mi-parcours étant au plus près d'autres revues littéraires comme L'Inconvénient et Contre-Jour. Si certaines trajectoires individuelles mériteraient d'être étudiées (celles de Yvon Rivard et de François Ricard entre autres), la comparaison que présente Nadon entre les lieux que sont Liberté et ces autres revues semble parfois artificielle. Certes, ailleurs on fait autre chose. Il n'est pas toujours facile de saisir à quoi sert cette comparaison qui ponctue les chapitres et clôt l'ouvrage au-delà d'une sorte de glorification camouflée de Liberté. On sort de cet ouvrage avec la claire compréhension d'une complexe filiation qui se joue à la fois sur les plans politique et esthétique, mais aussi à travers des discours explicites et implicites. L'héritage est lourd en effet dans l'histoire de Liberté et, s'il est vrai que ses fantômes sont partout, il est surtout de bon ton de dire qu'ils sont encombrants. Je ne le crois pas deux secondes. Car la beauté des filiations intellectuelles, c'est en effet qu'elles peuvent être choisies. 